\title{
High-resolution Mapping of Hyperglycemia-induced Gastric Slow Wave Dysrhythmias
}

\author{
Peng Du, ${ }^{1 *}$ Gregory 0'Grady, ${ }^{1,2}$ Niranchan Paskaranandavadivel, ${ }^{1}$ Shou-jiang Tang, ${ }^{3}$ Thomas Abell, ${ }^{4}$ and Leo K Cheng ${ }^{1,5}$ \\ ${ }^{\prime}$ Auckland Bioengineering Institute, University of Auckland, Auckland, New Zealand; ${ }^{2}$ Department of Surgery, University of Auckland, \\ Auckland, New Zealand; ${ }^{3}$ University of Mississippi Medical Center, Jackson, MS, USA; ${ }^{4}$ University of Louisville, Louisville, KY, USA; and \\ ${ }^{5}$ Department of Surgery, Vanderbilt University, Nashville, TN, USA
}

\section{Background/Aims}

It is now recognised that gastric dysrhythmias are best characterised by their spatial propagation pattern. Hyperglycemia is an important cause of gastric slow wave dysrhythmia, however, the spatiotemporal patterns of dysrhythmias in this context have not been investigated. This study aims to investigate the relationship between hyperglycemia and the patterns of dysrhythmias by employing high-resolution (multi-electrode) mapping simultaneously at the anterior and posterior gastric serosa.

\section{Methods}

High-resolution mapping $(8 \times 16$ electrodes per serosal) was performed in 4 anesthetized hounds. Baseline recordings $(21 \pm 8$ minutes) were followed by intravenous injection of glucagon ( $0.5 \mathrm{mg}$ per dose) and further recordings (59 \pm 15 minutes). Blood glucose levels were monitored manually using a glucose sensing kit at regular 5-minute intervals. Slow wave activation maps, amplitudes, velocity, anisotropic ratio, and frequency were calculated. Differences were compared between baseline and post glucagon injection.

\section{Results}

Baseline slow waves propagated symmetrically and antegrade. The blood glucose levels were increased by an average of $112 \%$ compared to the baseline by the end of the recordings. All subjects demonstrated elevated incidence of slow wave dysrhythmias following injection compared to the baseline ( $48 \pm 23 \%$ vs $6 \pm 4 \%, P<0.05)$. Dysrhythmias arose simultaneously or independently on anterior and posterior serosa. Spatial dysrhythmias occurred before and persisted after the onset and disappearance of temporal dysrhythmias.

\section{Conclusions}

Infusion of glucagon induced gastric slow wave dysrhythmias, which occurred across a heterogeneous range of patterns and frequencies. The spatial dysrhythmias of gastric slow waves were shown to be more prevalent and persisted over a longer period of time compared to the temporal dysrhythmias.

(J Neurogastroenterol Motil 2019;25:276-285)

\section{Key Words}

Electrophysiology; Gastrointestinal tract; Hyperglycemia; Interstitial cells of Cajal; Myoelectric complex, migrating

Received: November 13, 2018 Revised: December 27, 2018 Accepted: January 16, 2019

(a) This is an Open Access article distributed under the terms of the Creative Commons Attribution Non-Commercial License (http://creativecommons. org/licenses/by-nc/4.0) which permits unrestricted non-commercial use, distribution, and reproduction in any medium, provided the original work is properly cited.

${ }^{*}$ Correspondence: Peng Du, PhD

Auckland Bioengineering Institute, University of Auckland, Private Bag 92019, Auckland 1142, New Zealand Tel: +64-9-373-7599, Fax: +64-9-367-7157, E-mail: peng.du@auckland.ac.nz 


\section{Introduction}

Gastric slow waves regulate gastric motility. Slow waves are generated by the interstitial cells of Cajal (ICC), which entrain over a gradient of intrinsic frequencies to a single frequency in the stomach. ${ }^{1}$ Dysrhythmias of slow waves have been linked to a number of gastric disorders such as gastroparesis, chronic nausea, and vomiting, and functional dyspepisa. ${ }^{2-5}$ High-resolution (HR) mapping using spatially-dense electrode arrays to quantitatively describe the sequence of gastric slow waves in a number of animal species and humans. ${ }^{6-9}$ There are inter-species differences between the amplitude, velocity, frequency, and number of wavefronts in the stomach, but in general comparable activity occurs in large monogastric mammals studied. Gastric slow waves originate from a single pacemaker region along the greater curvature in the proximal stomach. Within the pacemaker region slow waves demonstrate a high degree of circumferentially-preferred anisotropic propagation before forming ring wavefronts in the mid-stomach corpus region. A transition to higher slow wave amplitude and velocity occurs in the distal stomach before the wavefront terminates at the pylorus. ${ }^{10}$

Hyperglycemia has been demonstrated to potentiate slow gastric emptying and onset of gastric dysrhythmias, ${ }^{11,12}$ and can induce abdominal symptoms of nausea, vomiting and pain. The degree of glycemic control in diabetes influences slow waves and motility. ${ }^{12-14}$ Previous slow wave recording studies involving hyperglycemia generally employed a glucose-clamping technique, ${ }^{12,13}$ or glucagon administeration. ${ }^{15}$ Evaluations of gastric dysrhythmias in response to hyperglycemia typically employed a limited number of electrodes, ${ }^{12,16}$ or cutaneous electrogastrography, ${ }^{17}$ and focused on frequency metrics. On the other hand, HR mapping has been used to define gastric slow wave responses to drugs such as opioids and vasopressin in spatiotemporal detail. ${ }^{18,19}$ One of the common findings from all of these studies is that spatial dysrhythmias could occur within normal frequency ranges, and therefore a temporal classification scheme alone could not describe the instances of slow wave dysrhythmias. Therefore, there is a need to understand the detailed spatial description of gastric dysrhythmias in relation to hyperglycemia, as similarly demonstrated previously in the intestines of a diabetic rat model. ${ }^{20}$

The purpose of this study is to gain better insight into the nature of hyperglycemia-induced gastric slow wave dysrhythmias under in vivo conditions. Hyperglycemia was induced by IV administration of glucagon, which is known to induce dysrhythmias, ${ }^{21}$ and HR mapping was applied concurrently on both the anterior and posterior serosal surfaces. The temporal and spatial components of slow wave dysrhythmias were assessed in relation to measurements of blood glucose (BG).

\section{Materials and Methods}

\section{Ethical Approval}

Ethical approval was granted by the University of Mississippi Medical Center Institutional Animal Care and Use Committee (No. 1265). The recording protocols were described previously ${ }^{19}$ and summarized here. All studies were conducted under general anesthesia, with continuous monitoring of vital signs. The anesthetic induction protocols included telezol (4.4 mg/kg; Tiletamine; Abbott Laboratories, North Chicago, IL, USA), domitor (0.08 mg/ kg; Medetomidine; Abbott Laboratories), burophanol (0.2 mg/kg; Stadol; Abbott Laboratories), and ketamine (10 mg/kg; Ketalar; Abbott Laboratories), and anesthesia was maintained using isoflurane (1-3\%; Forane; Abbott Laboratories) via IV. Vitals monitoring included heart rate, respiration rate, and oxygen saturation. Attention was given to the degree of muscle tone and any resistance to the ventilator. A midline laparotomy provided access the stomach, and the wound edges were approximated whenever possible to prevent gastric serosa from cooling and drying. The subject was euthanized after the conclusion of the experiment with an intracardiac injection of concentrated pentobarbital (Nembutal Sodium; Abbott Laboratories) to induce cardiac arrest, while under general anaesthesia.

\section{Animal Preparation and Recordings}

Experiments were performed on 4 hound-mix canines (20-23 $\mathrm{kg})$. The number of subjects was comparable to previous studies of similar designs. ${ }^{21,22}$ Glucagon was administered via intravenous infusion to the subjects. On average a $0.5 \mathrm{mg}$ dosage was administered at a time, and with BG levels measured at 5-minute intervals using a glucose meter kit (Accu-Chek; Roche, Rotkreuz, Switzerland) from the ear after the first dosage was administered.

Validated flexible printed circuit (FlexiMap, Auckland, New Zealand) recording arrays were used for mapping (inter-electrode spacing: 4 or $7.62 \mathrm{~mm}$ ). ${ }^{6}$ The electrode arrays were arranged into 2 "patches," with each patch containing 128 electrodes in a $16 \times 8$ configuration (a total of 256 electrodes), as shown in Figure 1. An anterior serosal patch was positioned over the antrum or lower corpus, and the second patch was placed on the posterior serosa in an approximately mirrored position. Attention was paid to ensure that the placements of the electrode arrays were reasonably consistent 

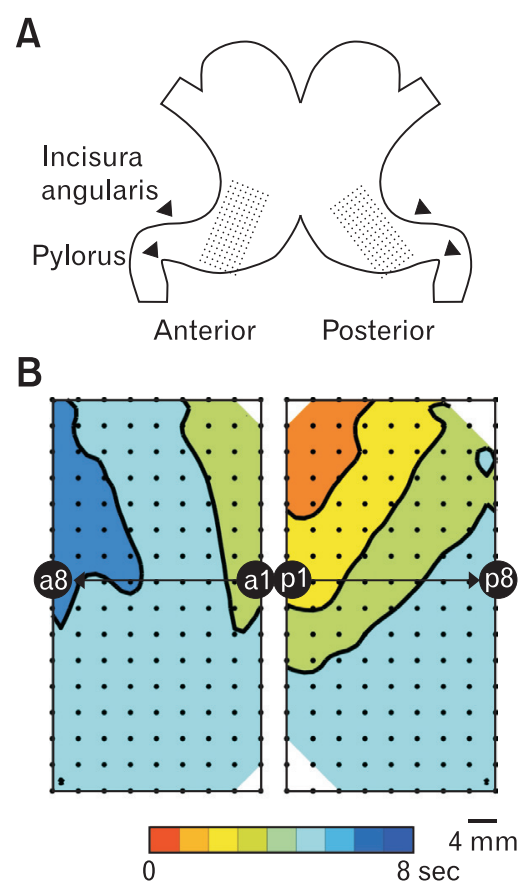

C

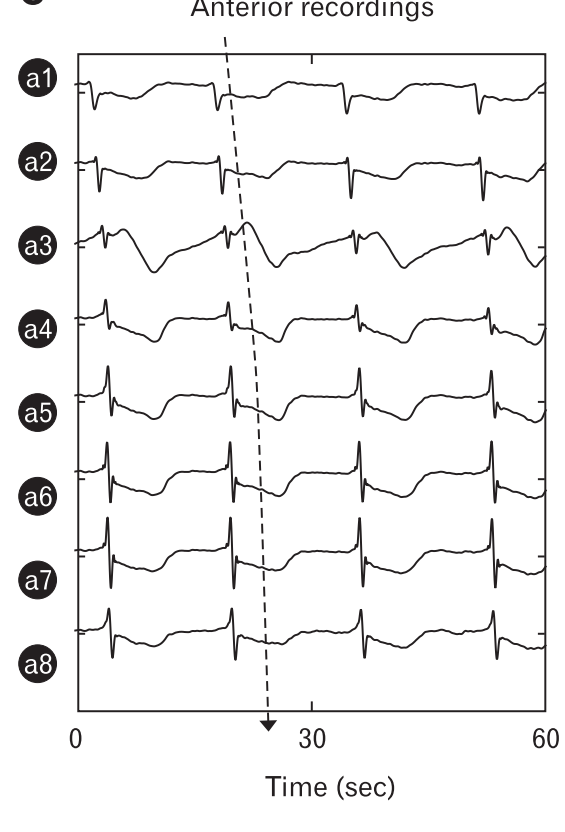

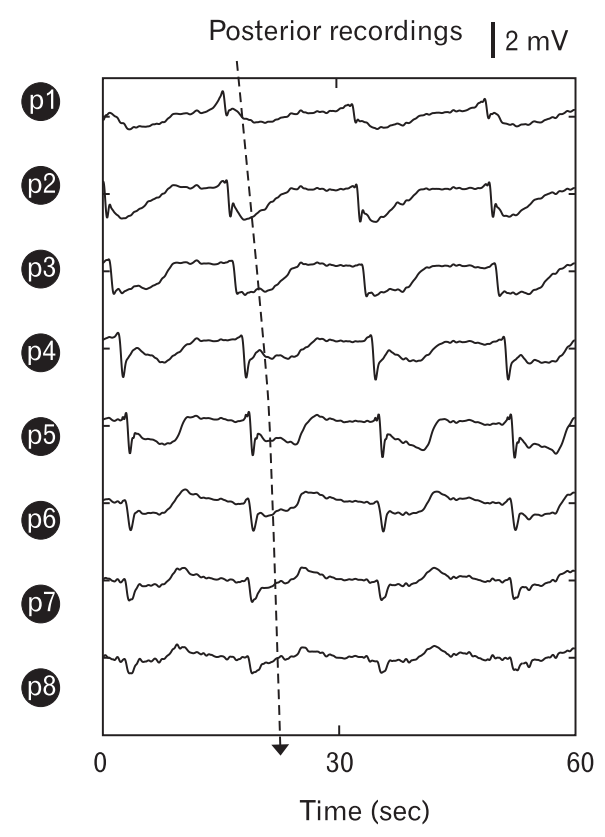

Fiğure 1. Setup and an example baseline recording prior to induced hyperglycemia. (A) Two arrays of electrodes $(8 \times 16 ; 4$ mm inter-electrode spacing) were placed on the anterior and posterior gastric serosa. (B) Activation maps were reconstructed from the recordings with each color band representing the area covered by 1 second of propagation. (C) Example electrograms selected from 8 anterior channels (a1-a8) and 8 posterior channels (p1-p8), with the dashed lines indicating the direction of propagation of a single cycle of slow waves.

relative to the major anatomical landmarks, such as the incisura and pylorus of the stomach, in a mirrored fashion between the 2 serosal surfaces. Warm saline-soaked gauze packs were gently placed over the electrodes to maintain gentle contact with the serosa. Signals were acquired at $512 \mathrm{~Hz}$ using a passive recording system (BioSemi, Amsterdam, the Netherlands), with the reference lead placed on the hind-leg.

\section{Data Processing}

All data analysis was performed in the gastrointestinal electrical mapping suite (GEMS) version 1.7.2. After down-sampling the data to $30 \mathrm{~Hz}$, and removal of baseline drift, a Savitzky-Golay filter was applied to the signals to remove high-frequency noise. ${ }^{24}$ Activation times of slow waves were detected and grouped using established automated algorithms. ${ }^{25,26}$ Manual review of the marked activation times was performed for all processed signals. The normal gastric slow wave pattern was defined as regular propagation in the antegrade direction towards the pylorus (Fig. 1B and 1C), whereas dysrhythmic events were defined as deviations of the normal propagation pattern, using criteria proposed in previous large animal studies. ${ }^{18,19,27}$ Previous studies have described an approximately 3-fold transition in the amplitude and velocity of slow waves in the distal antrum, ${ }^{8,19}$ and therefore all post-infusion recordings were compared specifically to the baseline recordings in each subject without moving the electrode arrays.

The dysrhythmias formed patterns with distinct circumferential and longitudinal components, eg, ectopic activity, the orthogonal velocity components were decomposed based on the velocity vector at the same electrode in the baseline recording. An "anisotropic ratio" (AR), ie, circumferential/longitudinal velocity was calculated for dysrhythmic episodes, which were determined relative to the baseline activities. ${ }^{27}$

\section{Statistical Methods}

Measured amplitude $(\mathrm{mV})$, velocity $(\mathrm{mm} / \mathrm{sec})$, interval $(\mathrm{sec})$, and frequency (cycles/min) values were defined for all detected slow wave events. Student's $t$ test was used to test statistical differences occurring during baseline and infusion of glucagon (significance threshold $P<0.05$ ). Mean values with standard deviation are reported as appropriate.

\section{Results}

Slow wave recordings with adequate coverage for mapping 
propagation were obtained from all subjects prior and following injection of glucagon. Direct HR mapping exhibited regular slow waves from both anterior and posterior surfaces of the stomach, the normal propagation pattern was comparable to previous HR mapping studies in canine subjects. ${ }^{8,19}$

The baseline recording was on average $21 \pm 8$ minutes. Gastric slow waves were recorded over an average duration of $59 \pm 15$ minutes following the induction of hyperglycemia. A total of 512 cycles of slow waves, on average $128 \pm 62$ cycles per subject, were analyzed following infusion of glucagon. Overall, the effects of hyperglycemia were significantly different compared to the baseline activity (Table). Over all analyzed cycles, on average, $48 \pm 23 \%$ of cycles showed dysrhythmias on spatiotemporal analysis, which was elevated compared to the baseline $(6 \pm 4 \%$ with abnormal propagation characteristics; $P<0.05)$.

The response to hyperglycemia is shown in Figure 2. The baseline BG level prior to glucagon infusion was $116 \pm 13 \mathrm{mg} / \mathrm{dL}$. Fifteen minutes after first glucagon infusion, the BG level rapidly increased to $225 \pm 166 \mathrm{mg} / \mathrm{dL}$ or $194 \%$ of the baseline value over the next 10 minutes, though significant variation in the BG levels were apparent within the first 15 minutes following glucagon induction. The average BG level increased to $246 \pm 35 \mathrm{mg} / \mathrm{dL}$ or $112 \%$ of the baseline value 50 minutes following glucagon induction of glucagon.

Slow wave amplitude was noted to be increased overall after administration of glucagon, by on average 1.1-1.7 $\mathrm{mV}$ compared to baseline $(P<0.0001)$. Glucagon also increased the velocity between $0.1-0.8 \mathrm{~mm} / \mathrm{sec}$ compared to baseline $(P$-value $<0.001)$. These changes in slow wave characteristics are consistent with past observations concerning dysrhythmias. ${ }^{28}$ Glucagon did not invoke an appreciable change in average frequency of gastric slow waves compared to baseline, when compared over the entire 50 minutes

Table. Definitions of Spatial Gastric Slow Wave Dysrhythmias

\begin{tabular}{lcc}
\hline \multicolumn{1}{c}{ Slow wave metrics } & Baseline & Glucagon \\
\hline Cycles analyzed (n) & $57 \pm 28$ & $128 \pm 62$ \\
Dysrhythmias (\% of waves) & $6 \pm 4$ & $48 \pm 23^{\mathrm{a}}$ \\
Amplitude (mV) & $2.6 \pm 0.6$ & $3.6 \pm 1.0^{\mathrm{a}}$ \\
Velocity (mm/sec) & $6.9 \pm 0.4$ & $7.3 \pm 1.3^{\mathrm{a}}$ \\
Anisotropic ratio & $0.0\left(2.2 \pm 0.8^{\mathrm{a}}\right)$ & $2.9 \pm 0.9^{\mathrm{a}}$ \\
Interval (sec) & $17.5 \pm 1.6$ & $23.0 \pm 5.6$ \\
Frequency (cycles/min) & $3.6 \pm 0.3$ & $3.3 \pm 0.6$ \\
\hline
\end{tabular}

${ }^{a}$ Indicates significance compared to the baseline data.

The anisotropic ratio of the spatial dysrhythmias during baseline is stated in the bracket.

Data are reported in mean \pm standard deviation. following first injection $(P>0.09)$. However, periods of both tachygastria and bradygastria were observed in all subjects (Fig. 2).

A notable feature was that dysrhythmia was evident as spatial propagation abnormalities as early as 7 minutes following injection of glucagon, and remained active until the end of the recording period (Fig. 2B), despite the amplitude, velocity, and frequency all returning to baseline. Frequencies in the tachygastria range were especially evident during a period of fluctuation from a slight depression at a rate of $-42 \mathrm{mg} / \mathrm{dL} / 5 \mathrm{~min}$ to recovery at a rate of 32 $\mathrm{mg} / \mathrm{dL} / 5 \mathrm{~min}$ of the BL measures between 15 to 30 minutes (Fig. 2A). The frequency of slow waves was elevated and exhibited larger fluctuations during this period compared to the slow waves outside

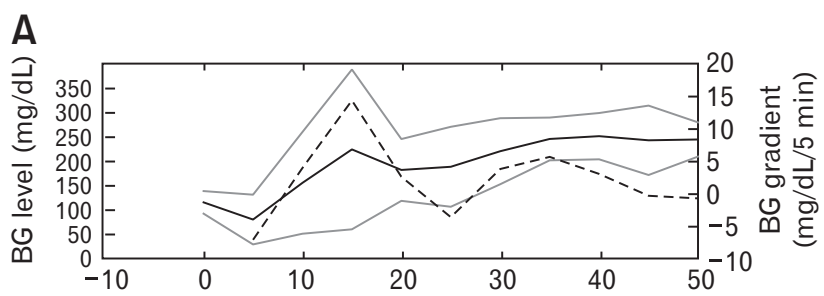

B

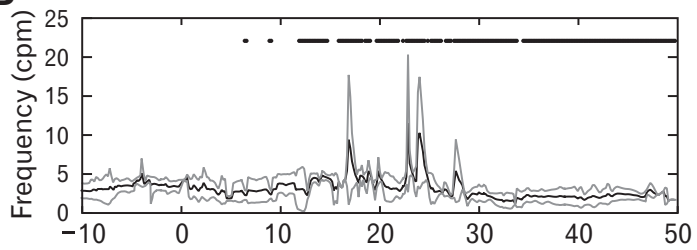

C
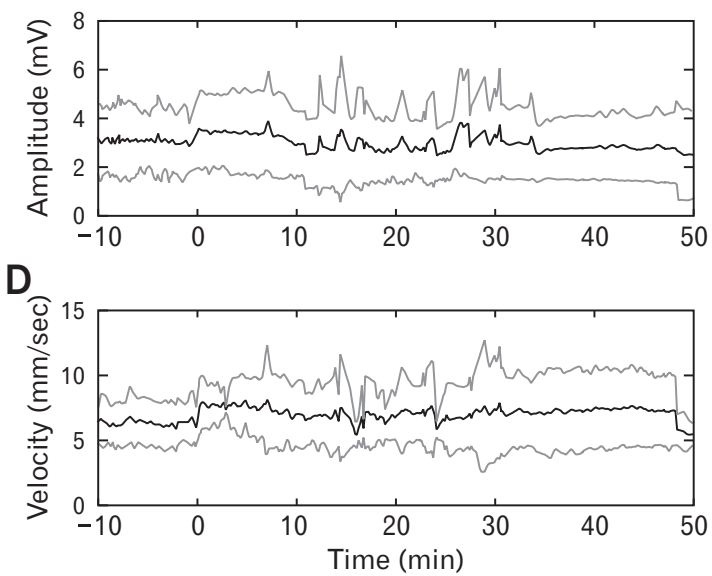

Figure 2. Summary of the effects of glucagon on gastric slow waves for all subjects. The gray lines represent standard deviations. (A) Blood glucose (BG) measurements following infusion of glucagon at $\mathrm{t}=0$ minute (solid line: average measure; dashed line: gradient). (B) Frequency of slow waves. The solid line/dot represents instances with spatial dysrhythmias in at least one subject. The dashed lines in all graphs represent one standard deviation from the average (solid line). (C) Amplitudes of slow waves. (D) Velocities of slow waves. 
this period (CI $[3.9,4.5]$ vs CI $[2.5,2.8] \mathrm{cpm} ; P<0.001)$.

In general, the dysrhythmias were highly dynamic, often transitioning from one type into another within a short interval. For example, a sustained re-entry accompanied with tachygastria of up to 30 seconds could be identified before reverting back to normal propagation following a period of quiescence (Fig. 3), which occurred in two-fourths subjects. Furthermore, the slow waves in the posterior surface were also found to propagate in the retrograde direction during the re-entry period in the anterior surface. The antegrade propagation that occurred following a period of quiescence was identical to the baseline data in Figure 1, though the frequency was significantly reduced to the bradygastria range.

Dysrhythmias resulting from ectopic distal pacemaker occurred in three-fourths subjects. In some instances (Fig. 4), repeated distal
A

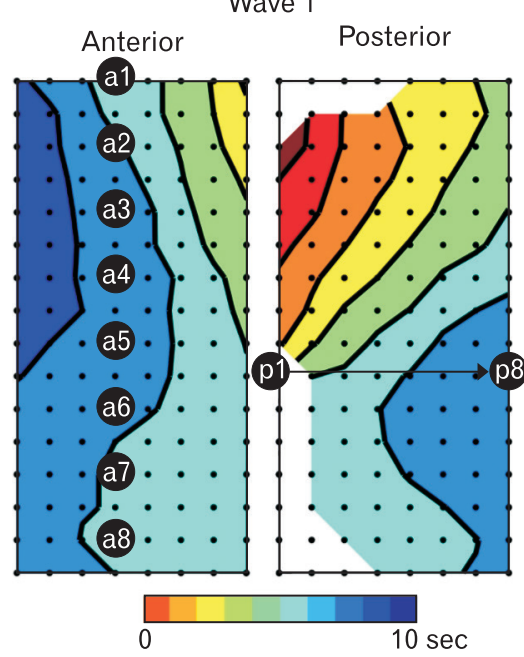

Wave 2

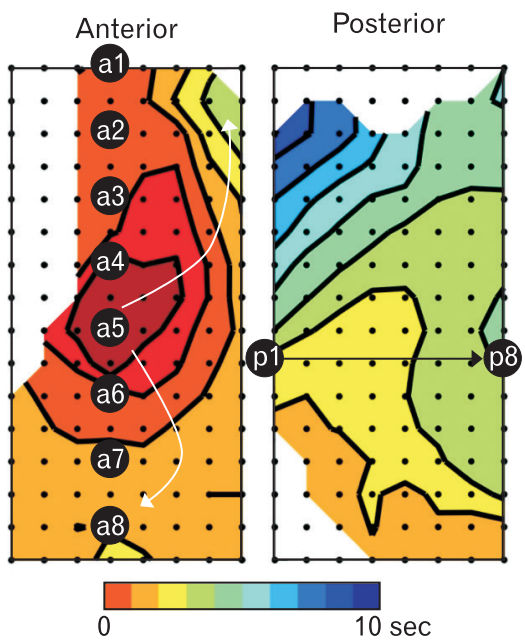

Wave 3

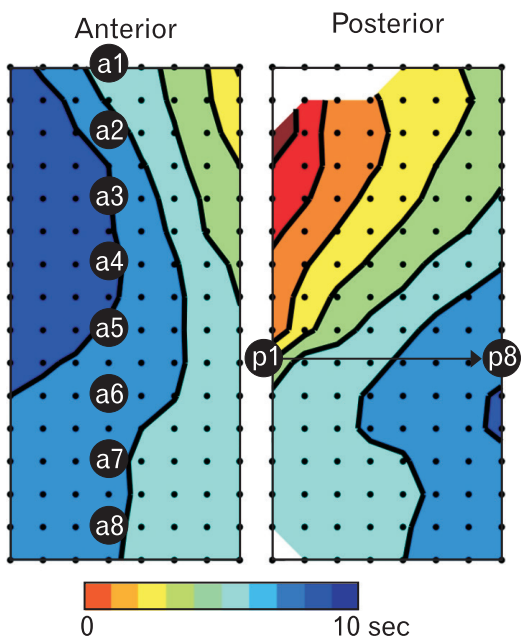

B
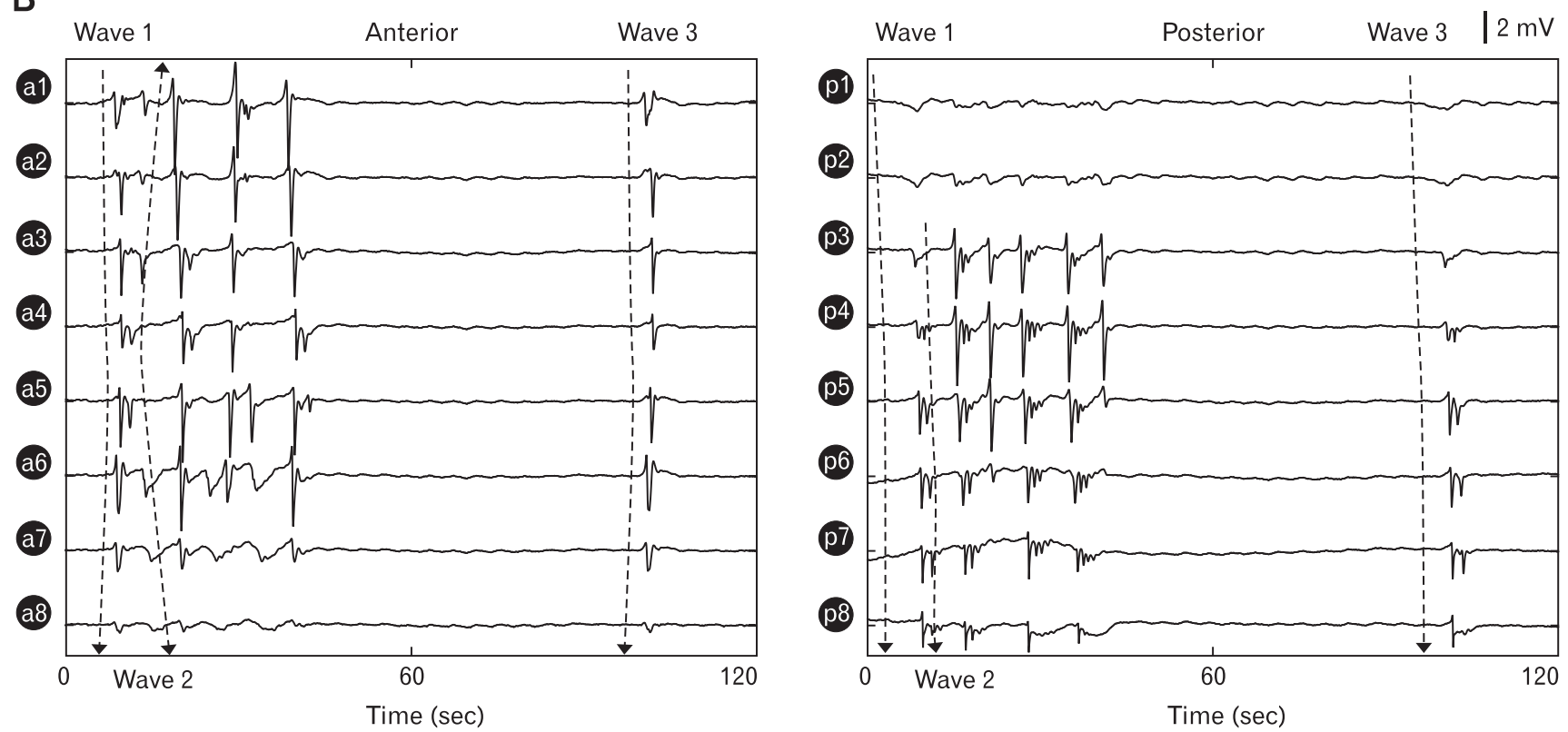

Figure 3. A dysrhythmic and tachygastria episode of gastric slow waves during hyperglycemia. (A) Activation maps of the start, mid and post dysrhythmia are shown. The first and third (waves 1 and 3) cycles both demonstrate the normal direction of propagation (Fig. 1A), whereas the second wave (wave 2) illustrates an episode of figure-of- 8 re-entry. (B) The selected electrograms demonstrated that tachygastria (up to 12 cpm) was associated with the period of re-entry (up to 30 seconds), followed by a 63 seconds of quiescence, before recovery back to the normal direction of propagation, with the exception of the double potentials in some of the posterior channels (p4-p7). 
A
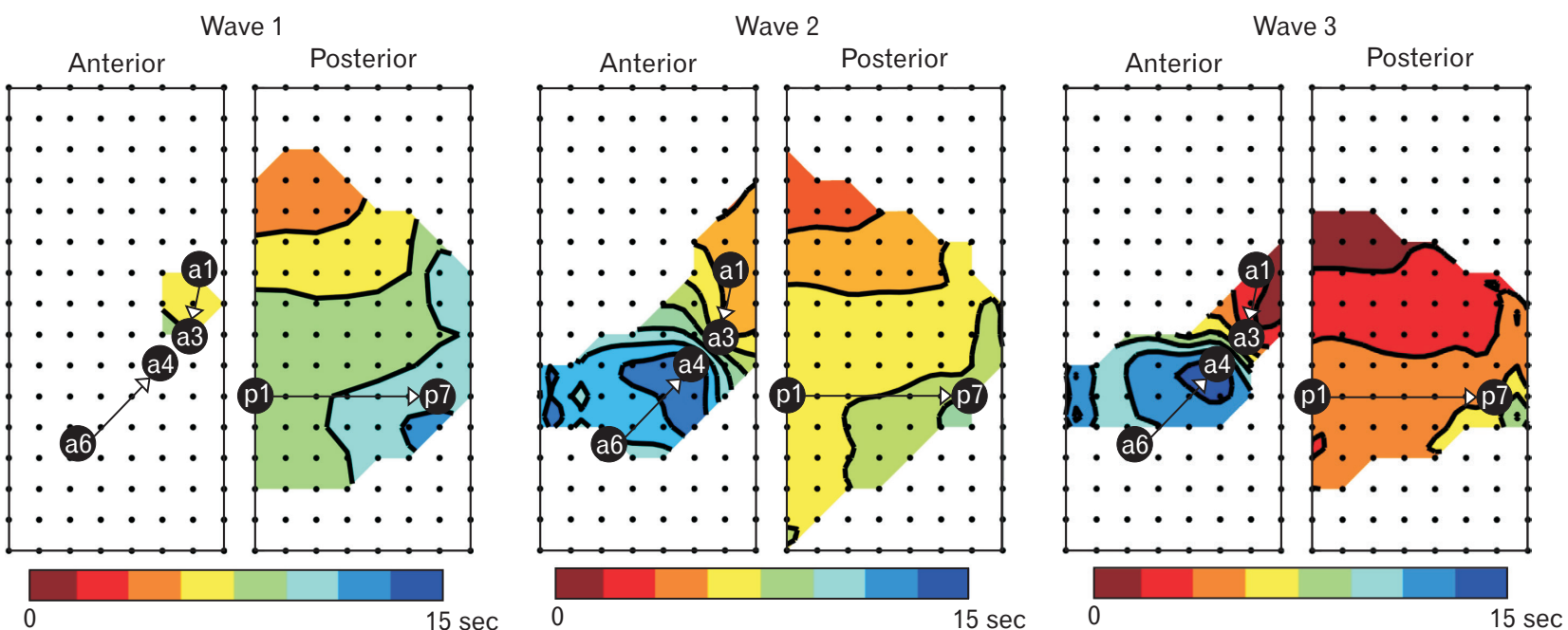

B

Anterior

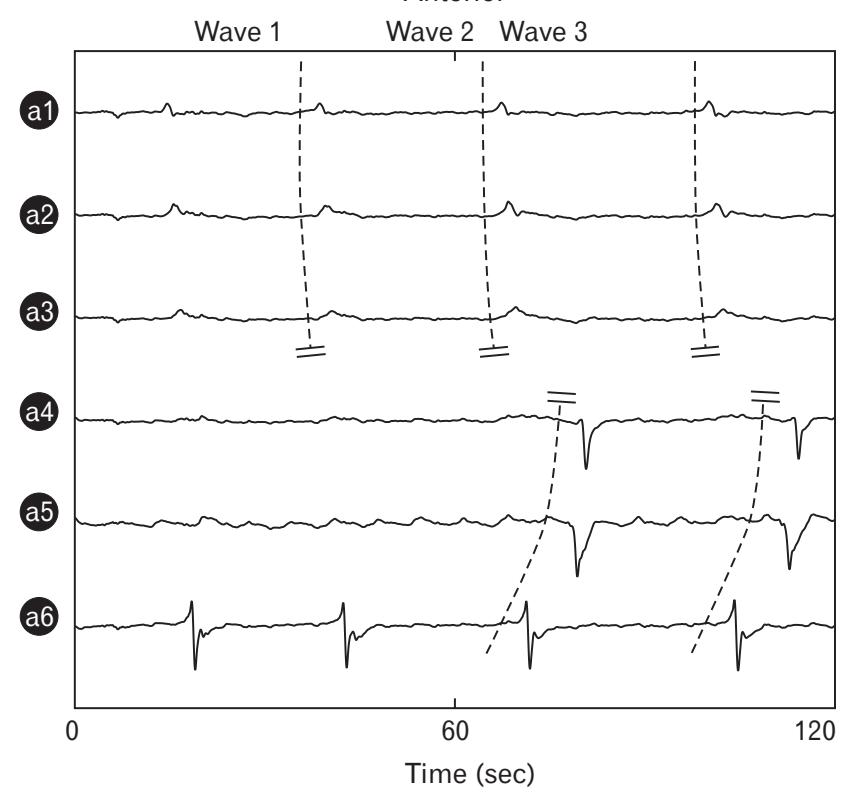

Posterior

$1 \mathrm{mV}$

Figure 4. Distal ectopic pacemaker followed by conduction block and then retrograde propagation. (A) Activation maps showing first conduction block of an antegrade wave (wave 1), and followed by clash due to retrograde propagation (waves 2-3) in the anterior serosa. (B) The electrograms demonstrate intermittent block to the retrograde propagation in the anterior serosa and uninterrupted antegrade propagation in the posterior serosa.

ectopic pacemaker was not able to invoke retrograde propagation in every cycle, due to conduction blocks. In this example, the average interval of the distal ectopic pacemaker and posterior slow waves were both $28.7 \pm 4.5$ seconds, while the average interval of the normal antegrade activity was $28.3 \pm 4.5$ seconds. In the cycles that the ectopic pacemaker was able to invoke retrograde propagation, it clashed with the proximal relatively lower amplitude antegrade propagation and was restricted in the anterior serosa only, while the posterior serosa maintained a normal propagation profile during the entire sequence.

Intermittent conduction block of antegrade propagation was observed following hyperglycemia induction in two-fourths subjects. The block covered at least $20 \mathrm{~mm}$ of the antrum in the antegrade direction (Fig. 5). While the frequency and propagation direction remained consistent throughout, the block occurred in about $70 \%$ of the cycles during the period of recordings. It could be possible that the block might be formed as a subsequent wavefront hitting the refractory tail of the preceding wavefront, as the frequen- 
A

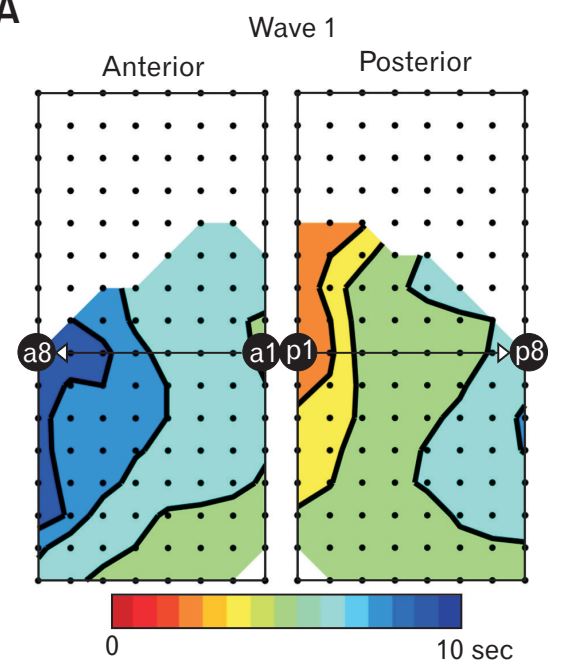

Wave 2

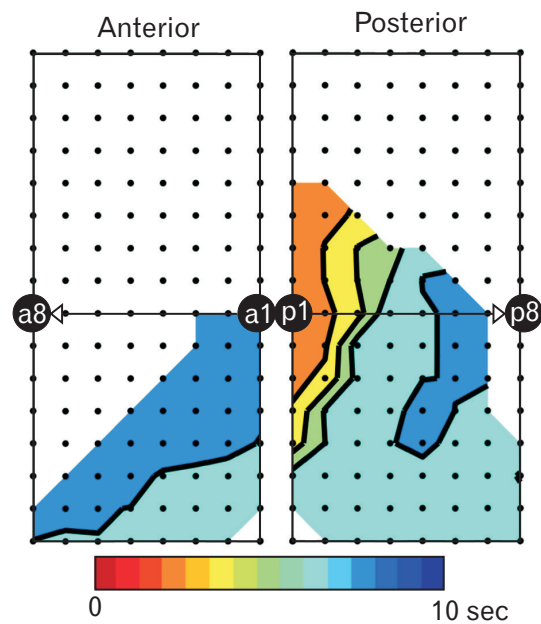

Wave 3

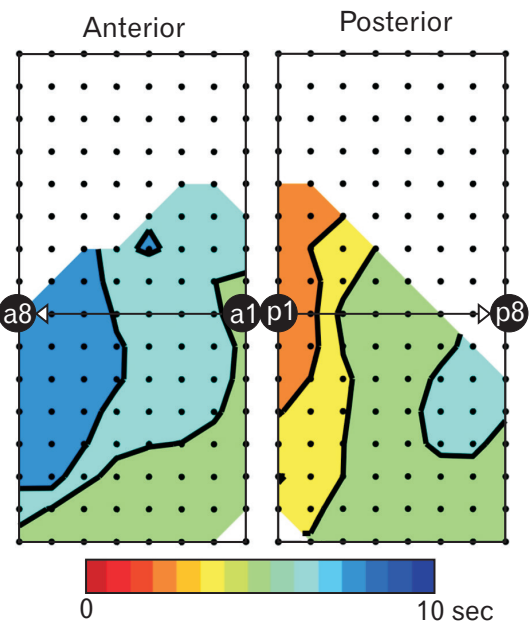

B

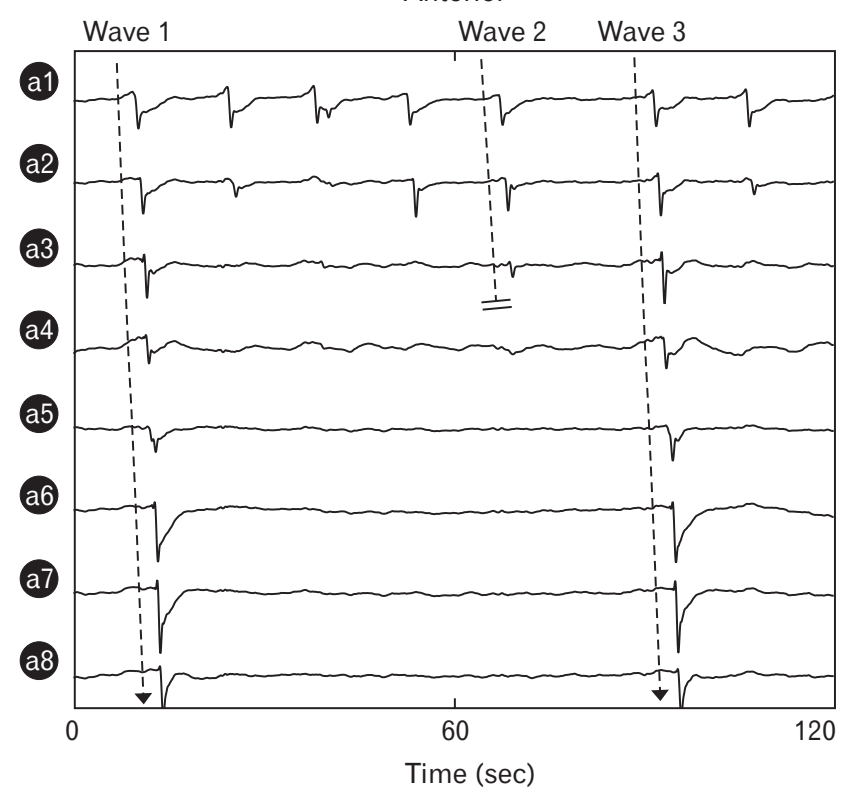

Posterior

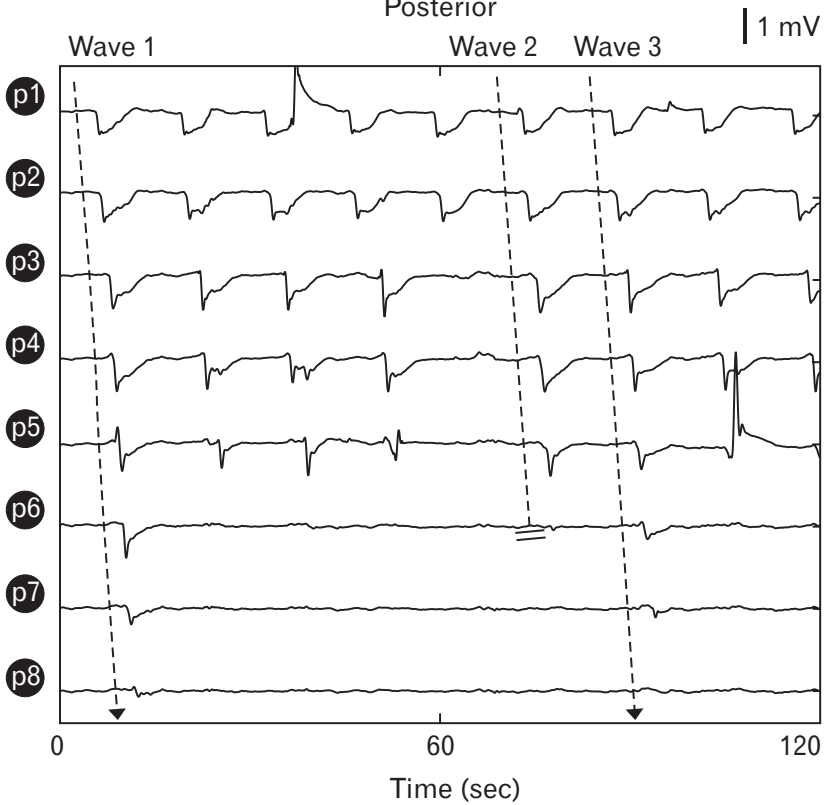

Figure 5. Intermittent block of antegrade propagation in the antrum. (A) Normal propagation wavefronts (waves 1 and 3 ) are interrupted by a block in the distal region (and wave 2) in between the 2 waves. (B) Electrograms demonstrating uninterrupted propagation to the pylorus and blocks. The region boundary of the block is illustrated by the double-dash line.

cy in the proximal region during the intermittent block period (13.7 \pm 0.4 seconds; $4.4 \pm 0.1 \mathrm{cpm}$ ) was higher than the baseline in this particular subject $(15.8 \pm 1.3$ seconds; $3.8 \pm 0.3 \mathrm{cpm} ; P$-values $<$ $0.0001)$.

\section{Discussion}

This study utilized HR mapping to spatiotemporally evaluate gastric slow wave dysrhythmias across both the anterior and poste- rior gastric serosal surfaces following acute induction of hyperglycemia in dogs. The main findings were: (1) all subjects developed sustained episodes of gastric dysrhythmias following hyperglycemia, (2) increase in variations of slow wave frequencies was strongly correlated with increase in the variations of amplitude and velocity of slow wave propagation, and (3) spatial patterns of dysrhythmias were observed prior to the onset of frequency changes and persisted after frequency had returned to baseline values. In summary, these findings demonstrate that hyperglycemia is associated with marked 
disturbances in slow wave behavior, and these are better characterized spatially than temporally.

Although an association between hyperglycemia and slow wave dysrhythmia has been well documented in a number of previous studies, all focused exclusively on a temporal or frequency-based analysis, ie, bradygastria or tachygastria. ${ }^{17,29}$ One of the key observations made in this study was that spatial dysrhythmias could occur prior to changes in temporal frequency, as well as persisting for a long time following the temporal dysrhythmias have subsided (Fig. 2). This finding is consistent with other recent HR mapping studies that have reported that spatially characterizing dysrhythmias offers a much superior sensitivity for detecting dysrhythmias than frequency-based changes alone.

A previous study also investigated the effects of vasopressin on gastric slow waves using the same methods used here. ${ }^{19}$ A number of observations could be made when comparing the effects of vasopressin to glucagon on gastric slow waves. Both drugs invoked similar levels of gastric slow wave dysrhythmias in terms of percentage of dysrhythmias, and amplitude and velocity changes. Although the results in this study suggest that vasopressin invoked more tachygastria episodes than glucagon, this could be attributed in part to the highly variable frequencies over a longer recording period in the glucagon study.

Hyperglycemia can also be induced by a dextrose-clamp protocol instead of glucagon administration. ${ }^{12}$ Intravenous dextrose and glucagon produce comparable effects when given in patients with hypoglycemia. ${ }^{30}$ In the present study, glucagon was administered as a bolus rather than infusion of dextrose at a sliding-scale value for the maintenance of $\mathrm{BG}$ levels, which required constant monitoring. It may be possible that changes in slow wave propagation could be associated with the rate of change of BG levels, ${ }^{31}$ in which case dextrose might be the better choice.

Rotor as a re-entry activity has been reported in a number of gastric dysrhythmia studies and in at least 2 different animal models. ${ }^{18,19}$ Consistent with these previous studies, the period of rotor was associated with elevated frequency into the tachygastria range, compared to the baseline frequency. The present study also demonstrated the long period of quiescence following the rotor activity before the propagation spontaneously reverted back to the normal propagation (Fig. 3). Interestingly, the activities during the rotor phase also demonstrated a series of rapid oscillations during the plateau and recovery phase of the slow waves. These oscillations were similar to the "spike activities" previously reported in animal models which were thought to be associated with the rapid discharge of calcium by gastric smooth muscle cells. ${ }^{32}$ However, in high-resolution extracellular mapping studies, spikes have been mostly observed in the intestines instead of the stomach, and have been shown to propagate independently from the slow waves. ${ }^{33}$ In the present study, spikes were only observed following slow waves and do not propagate independently, suggesting perhaps a more tightly regulated relationship between ICC and smooth muscles in the stomach.

One of the key observations from this study was the intermittent block of slow wave propagation into the antrum (Fig. 5). It could be possible that the block might be formed as a subsequent wavefront hitting the refractory tail of the preceding wavefront, as the frequency in the proximal region during the intermittent block period was higher than the baseline in this particular subject. The implication of this observation was that tachygastria could lead to premature termination of gastric slow waves in the distal stomach similar to those observed in the intestine. ${ }^{33,34}$ It could also be possible that the interval during which no antegrade wave propagate into the antrum could afford an ectopic activation to take place. Since antral contractions have been shown to be a major driver in the efficient mixing and emptying of gastric contents, ${ }^{10}$ a disruption to the slow waves in this region might have a significant impact on the normal digestive process.

A number of previous studies have reported clashing of gastric slow waves resulting from retrograde propagation, ${ }^{3,5,18,35}$ which has been demonstrated that the uncoupling could be restricted to one serosal surface only, while slow waves in the other serosal surface remained largely normal at the frequency of the normal antegrade propagating waves (Fig. 4). A previous study showed uncoupling and/or retrograde propagations could occur simultaneously in both serosal surfaces, ${ }^{19}$ suggesting an added level of complexity of spatial gastric dysrhythmias and demonstrating that comprehensively mapping slow waves at high spatiotemporal solutions across the whole stomach is optimal for dysrhythmia detection.

There are a number of specific limitations related to the present study. First, the size of the cohort in this short investigation was small, whereas investigations on a similar animal model were generally done in up to 6 subjects. ${ }^{21,22}$ It would be ideal if the monitoring could be done continuously during slow wave recordings over a larger portion of the stomach to align with the timings between BG levels and slow wave dysrhythmias. Finally, even though the recordings post-induction were on average 59 minutes long, the response was still acute and under anesthesia. It could be that the more significant effects would be more evident once the subjects have been subjected under repeated and chronic induced hyperglycemia or in the fed state. ${ }^{36}$

In summary, the present study quantitatively analyzed gastric 
dysrhythmias occurring during hyperglycemia in high spatiotemporal detail. Spatial dysrhythmias occurred earlier and persisted for longer than frequency disturbances. The source of dysrhythmias could be traced to either serosal surface, occurring either simultaneously or independently. The importance of spatial analysis of dysrhythmias rather than frequency-focused analyses is emphasized.

Financial support: This work and authors were funded by grants from the National Institute of Health (R01 DK64775), the Health Research Council of New Zealand, and the Medical Technologies Center of Research Excellence (MedTech CoRE) New Zealand. Peng Du is supported by the Rutherford Foundation Trust.

Conflicts of interest: Peng Du, Gregory O'Grady, Niranchan Paskaranandavadivel, and Leo K Cheng hold intellectual property in the field of gastrointestinal electrophysiology and are shareholders in FlexiMap Ltd. Thomas Abell is a former licensor, consultant, and investigator for Medtronic.

Author contributions: Peng Du performed data processing and analysis; Niranchan Paskaranandavadivel was responsible signal processing; Shou-jiang Tang, Thomas Abell, Gregory O'Grady, Leo K Cheng designed the experimental set and obtained experimental data; and Peng Du, Gregory O'Grady, Niranchan Paskaranandavadivel, Shou-jiang Tang, Thomas Abell, and Leo K Cheng were involved in the interpretation, writing and approval of the manuscript.

\section{References}

1. Huizinga JD, Lammers WJ. Gut peristalsis is governed by a multitude of cooperating mechanisms. Am J Physiol Gastrointest Liver Physiol 2009;296:G1-G8.

2. O'Grady G, Wang TH, Du P, Angeli T, Lammers WJ, Cheng LK. Recent progress in gastric arrhythmia: pathophysiology, clinical significance and future horizons. Clin Exp Pharmacol Physiol 2014;41:854-862.

3. O'Grady G, Angeli TR, Du P, et al. Abnormal initiation and conduction of slow-wave activity in gastroparesis, defined by high-resolution electrical mapping. Gastroenterology 2012;143:589-598, e3.

4. Lin X, Chen JZ. Abnormal gastric slow waves in patients with functional dyspepsia assessed by multichannel electrogastrography. Am J Physiol Gastrointest Liver Physiol. 2001;280:G1370-G1375.

5. Angeli TR, Cheng LK, Du P, et al. Loss of interstitial cells of cajal and patterns of gastric dysrhythmia in patients with chronic unexplained nausea and vomiting. Gastroenterology 2015;149:56-66, e5.

6. Du P, O'Grady G, Egbuji JU, et al. High-resolution mapping of in vivo gastrointestinal slow wave activity using flexible printed circuit board electrodes: methodology and validation. Ann Biomed Eng 2009;37:839-846.

7. Egbuji JU, O'grady G, Du P, et al. Origin, propagation and regional characteristics of porcine gastric slow wave activity determined by highresolution mapping. Neurogastroenterol motil 2010;22:e292-e300.

8. Lammers WJ, Ver Donck L, Stephen B, Smets D, Schuurkes JA. Origin and propagation of the slow wave in the canine stomach: the outlines of a gastric conduction system. Am J Physiol Gastrointest Liver Physiol 2009;296:G1200-G1210.

9. O'Grady G, Du P, Cheng LK, et al. Origin and propagation of human gastric slow-wave activity defined by high-resolution mapping. Am J Physiol Gastrointest Liver Physiol 2010;299:G585-G592.

10. Berry R, Miyagawa T, Paskaranandavadivel N, et al. Functional physiology of the human terminal antrum defined by high-resolution electrical mapping and computational modeling. Am J Physiol Gastrointest Liver Physiol 2016;311:G895-G902.

11. Plummer MP, Jones KL, Cousins CE, et al. Hyperglycemia potentiates the slowing of gastric emptying induced by exogenous GLP-1. Diabetes Care 2015;38:1123-1129.

12. Coleski R, Hasler WL. Coupling and propagation of normal and dysrhythmic gastric slow waves during acute hyperglycaemia in healthy humans. Neurogastroenterol Motil 2009;21:492-499, e1-2.

13. Fraser RJ, Horowitz M, Maddox AF, Harding PE, Chatterton BE, Dent J. Hyperglycaemia slows gastric emptying in type 1 (insulin-dependent) diabetes mellitus. Diabetologia 1990;33:675-680.

14. Horowitz M, Harding PE, Maddox AF, et al. Gastric and oesophageal emptying in patients with type 2 (non-insulin-dependent) diabetes mellitus. Diabetologia 1989;32:151-159.

15. Ouyang X, Li S, Foreman R, et al. Hyperglycemia-induced small intestinal dysrhythmias attributed to sympathovagal imbalance in normal and diabetic rats. Neurogastroenterol Motil 2015;27:406-415.

16. Chen JD, Qian L, Ouyang H, Yin J. Gastric electrical stimulation with short pulses reduces vomiting but not dysrhythmias in dogs. Gastroenterology 2003;124:401-409.

17. Abell TL, Malagelada JR. Glucagon-evoked gastric dysrhythmias in humans shown by an improved electrogastrographic technique. Gastroenterology 1985;88:1932-1940.

18. Lammers WJ, Ver Donck L, Stephen B, Smets D, Schuurkes JA. Focal activities and re-entrant propagations as mechanisms of gastric tachyarrhythmias. Gastroenterology 2008;135:1601-1611.

19. Du P, O’Grady G, Paskaranandavadivel N, Tang SJ, Abell T, Cheng LK. Simultaneous anterior and posterior serosal mapping of gastric slowwave dysrhythmias induced by vasopressin. Exp Physiol 2016;101:12061217.

20. Lammers WJ, Stephen BS, Karam SM. Slow wave dysrhythmias in the diabetic small intestine. Neurogastroenterol Motil 2015;27:1344.

21. Xu J, Chen JD. Effects of cyclooxygenase-2 inhibitor on glucagoninduced delayed gastric emptying and gastric dysrhythmia in dogs. Neurogastroenterol Motil 2007;19:144-151.

22. Xu J, Ross RA, McCallum RW, Chen JD. Two-channel gastric pacing with a novel implantable gastric pacemaker accelerates glucagon-induced delayed gastric emptying in dogs. Am J Surg 2008;195:122-129. 
23. Yassi R, O'Grady G, Paskaranandavadivel N, et al. The gastrointestinal electrical mapping suite (GEMS): software for analyzing and visualizing high-resolution (multi-electrode) recordings in spatiotemporal detail. BMC Gastroenterol 2012;12:60.

24. Paskaranandavadivel N, O'Grady G, Du P, Cheng LK. Comparison of filtering methods for extracellular gastric slow wave recordings. Neurogastroenterol Motil 2013;25:79-83.

25. Erickson JC, O'Grady G, Du P, Egbuji JU, Pullan AJ, Cheng LK. Automated gastric slow wave cycle partitioning and visualization for highresolution activation time maps. Ann Biomed Eng 2011;39:469-483.

26. Erickson JC, O'Grady G, Du P, et al. Falling-edge, variable threshold (FEVT) method for the automated detection of gastric slow wave events in high-resolution serosal electrode recordings. Ann Biomed Eng 2010;38:1511-1529.

27. Du P, O'Grady G, Paskaranandavadivel N, et al. Quantification of velocity anisotropy during gastric electrical arrhythmia. Conf Proc IEEE Eng Med Biol Soc 2011;2011:4402-4405.

28. O'Grady G, Du P, Paskaranandavadivel N, et al. Rapid high-amplitude circumferential slow wave propagation during normal gastric pacemaking and dysrhythmias. Neurogastroenterol Motil 2012;24:e299-e312.

29. Jebbink RJ, Samsom M, Bruijs PP, et al. Hyperglycemia induces abnormalities of gastric myoelectrical activity in patients with type I diabetes mellitus. Gastroenterology 1994;107:1390-1397.
30. Collier A, Steedman DJ, Patrick AW, et al. Comparison of intravenous glucagon and dextrose in treatment of severe hypoglycemia in an accident and emergency department. Diabetes Care 1987;10:712-715.

31. Hasler WL, Soudah HC, Dulai G, Owyang C. Mediation of hyperglycemia-evoked gastric slow-wave dysrhythmias by endogenous prostaglandins. Gastroenterology 1995; 108:727-736.

32. Stevens RJ, Weinert JS, Publicover NG. Visualization of origins and propagation of excitation in canine gastric smooth muscle. Am J Physiol 1999;277:C448-C460.

33. Lammers WJ, Faes C, Stephen B, Bijnens L, Ver Donck L, Schuurkes JA. Spatial determination of successive spikes in the isolated cat duodenum. Neurogastroenterol Motil 2004;16:775-783.

34. Parsons SP, Huizinga JD. Spatial noise in coupling strength and natural frequency within a pacemaker network; consequences for development of intestinal motor patterns according to a weakly coupled phase oscillator model. Front Neurosci 2016;10:19.

35. O'Grady G, Egbuji JU, Du P, et al. High-resolution spatial analysis of slow wave initiation and conduction in porcine gastric dysrhythmia. Neurogastroenterol Motil 2011;23:e345-e355.

36. Qian LW, Pasricha PJ, Chen JD. Origins and patterns of spontaneous and drug-induced canine gastric myoelectrical dysrhythmia. Dig Dis Sci 2003;48:508-515. 